\title{
Switching from tenofovir disoproxil fumarate to tenofovir alafenamide in antiretroviral regimens for virologically suppressed adults with HIV-1 infection: a randomised, active-controlled, multicentre, open-label, phase 3, non-inferiority study
}

\author{
Anthony Mills, Jose R Arribas, Jaime Andrade-Villanueva, Giovanni DiPerri, Jan Van Lunzen, Ellen Koenig, Richard Elion, Matthias Cavassini, \\ Jose Valdez Madruga, Jason Brunetta, David Shamblaw, Edwin Dejesus, Chloe Orkin, David A Wohl, Indira Brar, Jeffrey L Stephens, \\ Pierre-Marie Girard, Gregory Huhn, Andrew Plummer, Ya-Pei Liu, Andrew K Cheng, Scott McCallister, for the GS-US-292-0109 team
}

\section{Summary}

Background Antiretroviral regimens containing tenofovir disoproxil fumarate have been associated with renal toxicity and reduced bone mineral density. Tenofovir alafenamide is a novel tenofovir prodrug that reduces tenofovir plasma concentrations by $90 \%$, thereby decreasing off-target side-effects. We aimed to assess whether efficacy, safety, and tolerability were non-inferior in patients switched to a regimen containing tenofovir alafenamide versus in those remaining on one containing tenofovir disoproxil fumarate.

Methods In this randomised, actively controlled, multicentre, open-label, non-inferiority trial, we recruited HIV-1-infected adults from Gilead clinical studies at 168 sites in 19 countries. Patients were virologically suppressed (HIV-1 RNA $<50$ copies per $\mathrm{mL}$ ) with an estimated glomerular filtration rate of $50 \mathrm{~mL}$ per min or greater, and were taking one of four tenofovir disoproxil fumarate-containing regimens for at least 96 weeks before enrolment. With use of a third-party computer-generated sequence, patients were randomly assigned (2:1) to receive a once-a-day single-tablet containing elvitegravir $150 \mathrm{mg}$, cobicistat $150 \mathrm{mg}$, emtricitabine $200 \mathrm{mg}$, and tenofovir alafenamide $10 \mathrm{mg}$ (tenofovir alafenamide group) or to carry on taking one of four previous tenofovir disoproxil fumaratecontaining regimens (tenofovir disoproxil fumarate group) for $\mathbf{9 6}$ weeks. Randomisation was stratified by previous treatment regimen in blocks of six. Patients and treating physicians were not masked to the assigned study regimen; outcome assessors were masked until database lock. The primary endpoint was the proportion of patients who received at least one dose of study drug who had undetectable viral load (HIV-1 RNA $<50$ copies per $\mathrm{mL}$ ) at week 48 . The non-inferiority margin was $12 \%$. This study was registered with ClinicalTrials.gov, number NCT01815736.

Findings Between April 12, 2013 and April 3, 2014, we enrolled 1443 patients. 959 patients were randomly assigned to the tenofovir alafenamide group and 477 to the tenofovir disoproxil fumarate group. Viral suppression at week 48 was noted in $932(97 \%)$ patients assigned to the tenofovir alafenamide group and in $444(93 \%)$ assigned to the tenofovir disoproxil fumarate group (adjusted difference $4 \cdot 1 \%, 95 \%$ CI 1.6-6.7), with virological failure noted in ten and six patients, respectively. The number of adverse events was similar between the two groups, but study drug-related adverse events were more common in the tenofovir alafenamide group (204 patients [21\%] vs 76 [16\%]). Hip and spine bone mineral density and glomerular filtration were each significantly improved in patients in the tenofovir alafenamide group compared with those in the tenofovir disoproxil fumarate group.

Interpretation Switching to a tenofovir alafenamide-containing regimen from one containing tenofovir disoproxil fumarate was non-inferior for maintenance of viral suppression and led to improved bone mineral density and renal function. Longer term follow-up is needed to better understand the clinical impact of these changes.

Funding Gilead Sciences.

\section{Introduction}

Although most patients with HIV-1 infection have durable virological suppression with their first antiretroviral therapy (ART) regimen, switching to an alternative regimen could reduce pill burden or dosing frequency, improve adherence and tolerability, and reduce toxicity and costs. ${ }^{1}$ The clinical goal of patients switching a treatment regimen is to achieve one or more of these advantages, while maintaining virological suppression.

Tenofovir disoproxil fumarate is associated with excellent virological suppression, but some patients can develop clinically relevant nephrotoxicity over time, ${ }^{2}$ especially individuals with risk factors for renal disease. ${ }^{3}$ Some patients also have greater reductions in bone mineral density with tenofovir disoproxil fumarate than
Lancet Infect Dis 2016; 16: 43-52 Published Online November 2, 2015 http://dx.doi.org/10.1016/ S1473-3099(15)00348-5 See Comment page 3 Southern California Men's Medical Group, Los Angeles, CA, USA (A Mills MD); Hospital Universitario La Paz, IdiPAZ, Madrid, Spain (J R Arribas MD); Hospital Civil de Guadalajara CUCS, Universidad de Guadalajara, Guadalajara, Jal, Mexico

(Prof J Andrade-Villanueva MD); University Hospital Amadeo De Savoia, Turin, Italy (G DiPerri MD); University Medical Center, Hamburg Eppendorf Infectious Diseases Unit, Hamburg, Germany (Prof J Van Lunzen MD); Instituto Dominicano de Estudios Virologicos JDEZ, Santo Domingo, Dominican Republic (E Koenig MD); George Washington University School of Medicine, Washington, DC, USA (R Elion MD); Infectious Disease Service, Lausanne University Hospital and Lausanne University, Lausanne, Switzerland (M Cavassini MD);

Centro de Referenda e Treinamento Em DSTIAIDS, São Paulo, Brazil (J Valdez Madruga MD); University of Toronto, Toronto, ON, Canada (J Brunetta MD), La Playa Medical Group, San Diego, CA, USA

(D Shamblaw MD); Orlando Immunology Center, Orlando, FL, USA (E Dejesus MD); Barts and the London NHS Trust, London, UK (C Orkin MBBch); University of North Carolina at Chapel Hill, Chapel Hill, NC, USA (D A Wohl MD); Henry Ford Health System, Detroit, MI, 
USA (I BrarMD); Mercer University School of Medicine, Macon, GA, USA (J L Stephens MD); Infectious Diseases Department, Hopital Saint Antoine, Sorbonne Universites, Univ Paris and INSERM, Paris, France (Prof P-M Girard MD); Ruth M Rothstein Core Center, Cook County Hospital, Chicago, IL, USA (G Huhn MD); and Gilead Sciences, Foster City, CA, USA

(A Plummer BS, Y-P Liu PhD, A K Cheng MD, S McCallister MD)

Correspondence to: Dr Scott McCallister, Gilead Sciences, Foster City, CA 94404, USA scott.mccallister@gilead.com
Research in context

\section{Evidence before this study}

We searched PubMed for articles published in English between 1970 and June 15, 2015, about the side-effects of tenofovir disoproxil fumarate-containing treatment. Our search terms included "tenofovir", "creatinine", "proteinuria", "tubulopathy", and "bone mineral density". We reviewed about 50 articles. A phase 2 comparative study of tenofovir alafenamide versus tenofovir disoproxil fumarate as part of a single-tablet regimen with elvitegravir, cobicistat, and emtricitabine showed significant improvements in both renal and bone safety for patients in the tenofovir alafenamide group.

\section{Added value of this study}

This large study in virologically suppressed patients with HIV showed non-inferiority for elvitegravir, cobicistat, emtricitabine, and tenofovir alafenamide over tenofovir disoproxil fumarate-containing regimens on efficacy at week 48. Importantly, patients who switched to elvitegravir, cobicistat, emtricitabine, and tenofovir alafenamide after at least 96 weeks on a tenofovir disoproxil fumarate-containing regimen had significant improvements in proteinuria, specific proximal tubular proteinuria, bone mineral density, and diagnoses of osteopenia and osteoporosis. These data also show that post-switch improvements occur irrespective of the third drug used. The improvements in measures of renal and bone safety could represent a clinical confirmation of the effect of lower plasma tenofovir concentrations in patients switched to elvitegravir, cobicistat, emtricitabine, and tenofovir alafenamide. Moreover, both the speed of improvement in these objective tests (week 2 after the switch) and the magnitude of the improvement over time have not been previously described.

\section{Implications of all available evidence}

Elvitegravir, cobicistat, emtricitabine, and tenofovir alafenamide is a highly potent regimen that could be used in treatment-naive patients, with or without risk of renal or bone comorbidities, and it might be used as a strategic switch in patients using tenofovir-containing regimens who want to reduce their risk of tenofovir-related renal or bone toxicity. with other ART regimens, especially in the first 24 weeks of treatment. ${ }^{4,5}$ In two double-blind randomised controlled studies of tenofovir alafenamide versus tenofovir disoproxil fumarate (both added to elvitegravir, cobicistat, and emtricitabine) in the initial treatment of HIV-1 infection, more than $90 \%$ of patients receiving tenofovir alafenamide had virological suppression at week 48 , but renal and bone abnormalities were significantly reduced in patients allocated to tenofovir alafenamide compared with those allocated to tenofovir disoproxil fumarate. ${ }^{6}$

In this study, we aimed to evaluate whether efficacy, safety, and tolerability (including bone and renal outcomes) were non-inferior in patients switched to a single tablet regimen of tenofovir alafenamide plus elvitegravir, cobicistat, and emtricitabine, compared with patients who remained on one of four regimens containing tenofovir disoproxil fumarate.

\section{Methods}

\section{Study design and participants}

GS-US-292-0109 is a randomised, active-controlled, openlabel, multicentre, non-inferiority study. All patients were HIV-1-infected adults drawn from predefined Gilead clinical studies at 168 sites in 19 countries in North America, Europe, Latin America, Asia, and Australia in which patients were taking a tenofovir disoproxil fumarate-containing regimen recommended by treatment guidelines (appendix). ${ }^{1}$ All patients were virologically suppressed (HIV-1 RNA $<50$ copies per $\mathrm{mL}$ ), had an estimated glomerular filtration (eGFR) rate greater than $50 \mathrm{~mL}$ per min (calculated by the Cockcroft-Gault equation), and all had been on one of four regimens containing tenofovir disoproxil fumarate for at least 96 weeks-elvitegravir, cobicistat, emtricitabine, and tenofovir disoproxil fumarate; efavirenz, emtricitabine, and tenofovir disoproxil fumarate; cobicistat-boosted atazanavir plus emtricitabine and tenofovir disoproxil fumarate; or ritonavir-boosted atazanavir plus emtricitabine and tenofovir disoproxil fumarate. Written consent was obtained by investigators.

\section{Randomisation and masking}

We randomly assigned patients (2:1) to either switch to elvitegravir, cobicistat, emtricitabine, and tenofovir alafenamide (tenofovir alafenamide group) or to stay on their previous tenofovir disoproxil fumarate-containing regimen (tenofovir disoproxil fumarate group). A computer-generated allocation sequence was generated by Bracket (San Francisco, CA, USA) and randomisation was stratified by previous treatment regimen at screening in block sizes of six. Investigators determined eligibility, obtained the patient number, and received the treatment assignment based on the randomisation sequence. Patients and treating physicians were not masked to the assigned study regimen; outcome assessors were masked until database lock.

\section{Procedures}

All study drugs were prescribed to be taken orally once a day with food at about the same time each day for each patient, apart from efavirenz, emtricitabine, and tenofovir disoproxil fumarate, which was prescribed to be taken on an empty stomach at the hour of sleep.

Laboratory analyses (haematology, chemistry, and urinalysis; Covance Central Laboratory, Indianapolis, IN, 
USA), HIV-1 RNA (Taqman $2 \cdot 0$ assay [Roche, Pleasanton, CA, USA]), ${ }^{7}$ CD4 cell count, and complete or symptomdirected physical examinations were done at baseline and at all subsequent study visits (weeks 2, 4, 8, and 12, and then every 12 weeks up to week 96). Dual energy x-ray absorptiometry (DXA) scans for hip and spine bone mineral density were done before drug administration at baseline and then every 24 weeks throughout the study. All scans were blindly read by a centralised laboratory (BioClinica, Newtown, PA, USA). Blood and urine tests for renal and bone biomarkers were done at baseline, weeks 2, 4, 12, 24, and 48 and at early study drug discontinuation visit. Kidney function tests included serum creatinine, eGFR by Cockcroft Gault equation $\left(\mathrm{eGFR}_{\mathrm{CG}}\right)$, and eGFR by Chronic Kidney Disease Epidemiology Collaboration and creatinine method $\left(\mathrm{eGFR}_{\text {CKD-EPI }}\right) .^{8-11}$ Proteinuria was assessed qualitatively by dipstick urinalysis, quantitatively by urine protein to creatinine ratio, urine albumin to creatinine ratio, and specific proximal renal tubular proteinuria (retinol binding protein to creatinine ratio, $\beta 2$-microglobulin to creatinine ratio); renal function was also assessed by renal tubular maximum reabsorption rate of phosphate to the glomerular filtration rate, fractional excretion of phosphate, and fractional excretion of uric acid. Adverse events and concomitant drugs were assessed at each visit. At 96 weeks, all patients had the option to switch to open-label elvitegravir, cobicistat, emtricitabine, and tenofovir alafenamide.

\section{Outcomes}

The primary efficacy endpoint was the proportion of patients with HIV-1 RNA of less than 50 copies per mL at week 48 after randomisation with use of the US Food and Drug Administration (FDA)-defined snapshot algorithm. ${ }^{12}$ Prespecified secondary outcomes were percentage change in hip and spine bone mineral density, change in serum creatinine, and change in efavirenz-related symptom score at week 48. Efficacy, safety, and tolerability were also assessed up to 96 weeks. Safety evaluations included standard laboratory testing and adverse event, coded with version 17.0 of the Medical Dictionary for Regulatory Activities (MedDRA). Pharmacokinetic testing, quality of life questionnaires, ${ }^{13-15}$ an assessment of CNS-related symptoms (patients on prior efavirenz only), ${ }^{16-18}$ and FRAX evaluation ${ }^{19}$ were used in the study and will be reported elsewhere.

\section{Statistical analysis}

The primary efficacy endpoint was tested with a conventional $95 \% \mathrm{CI}$ approach for the difference in response rates (tenofovir alafenamide group minus tenofovir disoproxil fumarate group); the lower bound of the $95 \%$ CI was assessed using a non-inferiority margin of $12 \%$. All patients who were randomised and had received at least one dose of the study drug were included in the primary endpoint analysis (full analysis set).
Assuming a $90 \%$ response rate for both groups at week 48 , the power to establish non-inferiority ranged from $90 \%$ to $99 \%$ depending on the number of participating patients. It was intended to recruit 1500 patients from the six prior studies and 1436 were randomised, the study had greater than $99 \%$ power to establish non-inferiority at week 48 ; the significance level of the test was at a one-sided $\alpha$ of 0.025 .

We summarise baseline characteristics with descriptive statistics. For categorical data, we calculated $\mathrm{p}$ values from the Cochran-Mantel-Haenszel test (general association statistic was used for nominal data, row mean scores differ statistic was used for ordinal data). For continuous data, $\mathrm{p}$ value was from the two-sided Wilcoxon rank sum test.

For the primary endpoint, we constructed the percentage differences and the associated 95\% CI with Mantel-Haenszel proportion, ${ }^{20}$ stratified by previous treatment regimen. In the snapshot analysis of full analysis set, patients with HIV-1 RNA less than 50 copies per $\mathrm{mL}$ in the week 48 window (between days 294 and 377) were classified as a virological success. We classified the following as virological failure: patients with HIV-1 RNA of 50 or more copies per $\mathrm{mL}$, patients with missing HIV-1 RNA data in the week 48 window, patients who discontinued study drug before week 48 , or patients who changed treatment before week 48. A prespecified superiority test was also done using the same 95\% CI constructed for evaluating non-inferiority; if the lower bound of the $95 \%$ CI was greater than 0 , superiority would be concluded.

We also analysed the primary efficacy endpoint by classifying missing data as failure, classifying missing data as excluded, and using the per-protocol analysis set; patients were included in the per-protocol if they received at least one dose of study drug unless they discontinued before week 48 or had an adherence rate in the lowest 2.5 percentile. We repeated the primary endpoint analysis in the full analysis with a cutoff of less than 20 copies per $\mathrm{mL}$. Changes in CD4 cell count from baseline to week 48 in the full analysis and per-protocol sets were summarised by treatment group with descriptive statistics using on-treatment data.

We summarised safety data in the full analysis set with descriptive statistics. For all secondary endpoint analyses, we pooled patients taking ritonavir-boosted atazanavir, emtricitabine, and tenofovir disoproxil fumarate and cobicistat-boosted atazanavir, emtricitabine, and tenofovir disoproxil fumarate to reduce non-relevant subgrouping. For key secondary endpoint safety data (percentage change in hip and spine bone mineral density, change from baseline in serum creatinine), we did prespecified between-group comparisons at week 48 with descriptive statistical testing. The difference in percentage change from baseline for spine and hip bone mineral density was constructed with an ANOVA model including previous treatment regimen as a fixed effect. 
We summarise the difference in serum creatinine separately for patients previously on efavirenz, emtricitabine, and tenofovir disoproxil fumarate and for patients previously on cobicistat or ritonavir boosted regimens; this was constructed with an ANCOVA model including previous treatment regimen as a fixed effect and with baseline serum creatinine as a covariate. For percentage change from baseline in proteinuria, the differences between treatment groups were analysed using a Van Elteren test stratified by previous treatment regimen. For other continuous laboratory test results, Wilcoxon rank sum testing was used. To control for the overall type 1 error rate in the primary efficacy endpoint assessment and the four key safety endpoints, we did hypothesis testing in sequential order with a protocolspecified fallback procedure. We used SAS version 9.2 for all analyses.

An independent data monitoring committee was used. This study was registered with ClinicalTrials.gov, number NCT01815736.

\section{Role of the funding source}

Gilead Sciences funded the study, collected, and analysed the data, interpreted the results, and helped to write the report. AM had full access to the data, interpreted the results, and helped to write the report. All authors had full access to the data, could request additional analyses, and could provide input into the interpretation of results. $\mathrm{AM}, \mathrm{AKC}$, and SM made the decision to submit the manuscript for publication.

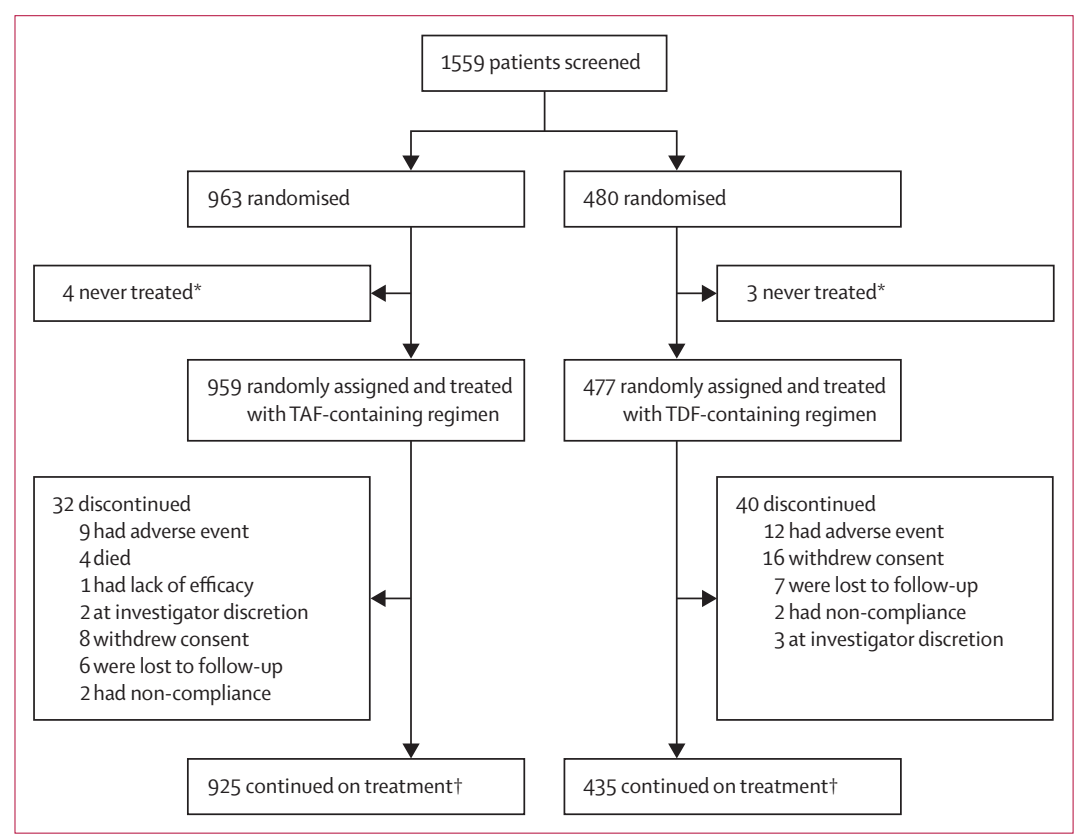

Figure 1: Study profile

*Four patients withdrew consent, two withdrew by investigator discretion, and one was lost to follow-up. †Four patients (two for each group) completed the randomised phase of the study and are now past week 96 TDF=tenofovir disoproxil fumarate. TAF=tenofovir alafenamide.

\section{Results}

Between April 12, 2013 and April 3, 2014, we randomly assigned 1443 patients; 1436 received at least one dose of study drug (figure 1). Of these 1436 patients, regimens received pre-randomisation were: elvitegravir $150 \mathrm{mg}$, cobicistat $150 \mathrm{mg}$, emtricitabine $200 \mathrm{mg}$, and tenofovir disoproxil fumarate $300 \mathrm{mg}(\mathrm{n}=459)$; efavirenz $600 \mathrm{mg}$ emtricitabine $200 \mathrm{mg}$, and tenofovir disoproxil fumarate $300 \mathrm{mg}(\mathrm{n}=376)$; and cobicistat-boosted $(\mathrm{n}=216)$ or ritonavir-boosted $(\mathrm{n}=385)$ atazanavir $300 \mathrm{mg}$, emtricitabine $200 \mathrm{mg}$, and tenofovir disoproxil fumarate $300 \mathrm{mg}$. We randomly assigned 959 of these patients to switch to elvitegravir $150 \mathrm{mg}$, cobicistat $150 \mathrm{mg}$, emtricitabine $200 \mathrm{mg}$, and tenofovir alafenamide $10 \mathrm{mg}$ (tenofovir alafenamide group). The remaining 477 patients remained on their previous tenofovir disoproxil fumaratecontaining regimen (tenofovir disoproxil fumarate group). 153 patients remained on elvitegravir, cobicistat, emtricitabine, and tenofovir disoproxil fumarate; 125 remained on efavirenz, emtricitabine, and tenofovir disoproxil fumarate; 69 remained on cobicistat-boosted atazanavir, emtricitabine, and tenofovir disoproxil fumarate; and 130 remained on ritonavir-boosted atazanavir, emtricitabine, and tenofovir disoproxil fumarate.

Baseline demographics were balanced between the two treatment groups with the exception of ethnic origin; more patients in the tenofovir alafenamide group than in the tenofovir disoproxil fumarate group reported Hispanic or Latino ethnic origin ( $\mathrm{p}=0.0006$; table 1$)$. The two groups had similar probability of 10 year major osteoporotic fracture by FRAX estimation. ${ }^{19}$

At week 48, 932 (97\%) of 959 patients in the tenofovir alafenamide group had virological success (HIV-1 RNA $<50$ copies per $\mathrm{mL}$ ), compared with 444 (93\%) of 477 patients in the tenofovir disoproxil fumarate group $(\mathrm{p}=0.0002)$. The difference in percentage, adjusted by previous treatment regimen, was $4 \cdot 1 \%$ (95\% CI 1.6-6 -7). Because the lower bound of this $95 \%$ CI was greater than the prespecified $12 \%$ margin, the tenofovir alafenamide regimen was non-inferior to the tenofovir disoproxil fumarate-containing regimens. The lower bound was also greater than zero, and therefore the tenofovir alafenamide regimen was statistically superior to the tenofovir disoproxil fumarate-containing regimens.

Virological failures were similar between groups with ten $(1 \%)$ in the tenofovir alafenamide group and six $(1 \%)$ in the tenofovir disoproxil fumarate group (detailed in appendix). One $(<1 \%)$ patient in the tenofovir alafenamide group had virological failure with genotypic resistance to a component of the treatment regimen. This patient had virological failure at week 8 with development of a Met184Ile/Met mixture; 4 weeks later this patient resuppressed to HIV-1 RNA less than 50 copies per $\mathrm{mL}$ without a change of regimen. More patients discontinued treatment for non-virological reasons in the tenofovir disoproxil fumarate group than in the tenofovir alafenamide group (appendix) 
In the per-protocol analysis, 913 (99\%) of 921 patients in the tenofovir alafenamide group and 435 (99\%) of 440 patients in the tenofovir disoproxil fumarate group had virological success (percentage difference $0 \cdot 3 \%$ ). Results from the missing equals failure and missing equals excluded analyses were consistent with the primary endpoint (appendix). Rates of virological success in the full analysis set were similar for subgroups of age, sex, race, and geographic region (appendix). Virological success at a cutoff of HIV-1 RNA less than 20 copies per $\mathrm{mL}$ was noted in 897 (94\%) of 959 patients in the tenofovir alafenamide group and $431(90 \%)$ of 477 patients in the tenofovir disoproxil fumarate group (adjusted percentage difference $3 \cdot 2 \%, 95 \%$ CI $0 \cdot 1-6 \cdot 3$ ).

When the primary endpoint was calculated stratified by previous treatment regimens, the proportion of patients with virological control varied. Of patients previously on elvitegravir, cobicistat, emtricitabine, and tenofovir disoproxil fumarate before randomisation, 98\% (301 of 306) who switched to tenofovir alafenamide maintained virological control, compared with $97 \%$ (149 of 153) who continued their regimen (percentage difference $1.0 \%$; $95 \%$ CI -1.9 to 3.9$)$. Of patients previously on efavirenz, emtricitabine, and tenofovir disoproxil fumarate before randomisation, $96 \%$ (241 of 251) who switched to tenofovir alafenamide maintained virological control compared with $90 \%$ (112 of 125 ) who continued their regimen (percentage difference $6 \cdot 4 \%, 95 \%$ CI $0 \cdot 5-12 \cdot 3$ ). Of patients on boosted atazanavir plus emtricitabine and tenofovir disoproxil fumarate before randomisation, $97 \%$ (390 of 402) who switched to tenofovir alafenamide maintained virological control, compared with 92\% (183 of 199) who continued their regimen (percentage difference, 95\% CI 0.9-9.2).

Mean changes in CD4 cell counts were similar between groups: increase of 35 cells per $\mu \mathrm{L}$ (SD 165) for the tenofovir alafenamide group versus increase of 24 cells per $\mu \mathrm{L}$ (SD 156) for the tenofovir disoproxil fumarate group.

Mean bone mineral density at the hip and spine increased in the tenofovir alafenamide group while remaining stable or decreasing at both sites in the tenofovir disoproxil fumarate group ( $<<0 \cdot 0001$; table 2 ). Hip and spine bone mineral density improved in patients assigned to the tenofovir alafenamide group compared with the tenofovir disoproxil fumarate group, irrespective of previous treatment. T-score bone mineral density for both hip and spine increased in patients assigned to the tenofovir alafenamide group, while remaining stable in those who continued their initial tenofovir disoproxil fumarate regimen (table 2).

In a subset of patients who received 144 weeks of ritonavir-boosted atazanavir plus emtricitabine and tenofovir disoproxil fumarate in study GS-US-236-0103, mean percentage reduction in hip bone mineral density from baseline was $4.58 \%$ (SD 4.17). ${ }^{21}$ In this study, patients in this subset randomly assigned to the tenofovir alafenamide group $(\mathrm{n}=41)$ had improved hip bone mineral density by $1.35 \%$ (SD 3.07 ) whereas those remaining on prior treatment $(n=13)$ had further reduction of $0.77 \%$ (SD 2.45). In this same subset, the mean percentage reduction in spine BMD in the previous

\begin{tabular}{|c|c|c|}
\hline & $\begin{array}{l}\text { Tenofovir alafenamide } \\
\text { group }(n=959)\end{array}$ & $\begin{array}{l}\text { Tenofovir disoproxil } \\
\text { fumarate group }(n=477)\end{array}$ \\
\hline Age (years) & $41(33-48)$ & $40(33-48)$ \\
\hline Men & $856(89 \%)$ & $427(90 \%)$ \\
\hline Women & $103(11 \%)$ & $50(10 \%)$ \\
\hline \multicolumn{3}{|l|}{ Race } \\
\hline Native American & $5(1 \%)$ & $2(<1 \%)$ \\
\hline Asian & $59(6 \%)$ & $35(7 \%)$ \\
\hline Black & $169(18 \%)$ & $102(21 \%)$ \\
\hline Native Hawaiian & $6(1 \%)$ & $1(<1 \%)$ \\
\hline White & $651(68 \%)$ & $314(66 \%)$ \\
\hline \multicolumn{3}{|l|}{ Ethnic origin } \\
\hline Hispanic or Latino & $248(26 \%)$ & $82(17 \%)$ \\
\hline Baseline body-mass index $\left(\mathrm{kg} / \mathrm{m}^{2}\right)$ & $25 \cdot 8(23 \cdot 1-29 \cdot 1)$ & $26 \cdot 1(23 \cdot 1-29 \cdot 4)$ \\
\hline HIV-1 RNA $<50$ copies per $\mathrm{mL}$ & $943(98 \%)$ & $466(98 \%)$ \\
\hline CD4 count (cells per $\mu \mathrm{L}$ ) & $675(520-833)$ & $662(525-831)$ \\
\hline \multicolumn{3}{|l|}{ CD4 count categories } \\
\hline$<50$ cells per $\mu \mathrm{L}$ & 0 & 0 \\
\hline 50 to $<200$ cells per $\mu \mathrm{L}$ & $5(1 \%)$ & $4(1 \%)$ \\
\hline 200 to $<350$ cells per $\mu \mathrm{L}$ & $54(6 \%)$ & $25(5 \%)$ \\
\hline 350 to $<500$ cells per $\mu \mathrm{L}$ & $151(16 \%)$ & $70(15 \%)$ \\
\hline 500 cells per $\mu \mathrm{L}$ & $749(78 \%)$ & $378(79 \%)$ \\
\hline \multicolumn{3}{|l|}{ Mode of infection (HIV risk factors)* } \\
\hline Heterosexual sex & $216(23 \%)$ & $101(21 \%)$ \\
\hline Homosexual sex & $753(79 \%)$ & $375(79 \%)$ \\
\hline Intravenous drug use & $9(1 \%)$ & $5(1 \%)$ \\
\hline Transfusion & $2(<1 \%)$ & $2(<1 \%)$ \\
\hline Vertical transmission & 0 & 0 \\
\hline Other & $8(1 \%)$ & $7(1 \%)$ \\
\hline Unknown & $17(2 \%)$ & $12(3 \%)$ \\
\hline
\end{tabular}

Estimated glomerular filtration rate by Cockcroft-Gault (CG) formula (mL per min)

$\begin{array}{lll}\text { Mean (SD) } & 111.9(33.38) & 112.1(32.69) \\ \text { Median } & 105.7(89.4-126.0) & 107.7(88.7-128.2)\end{array}$

Estimated glomerular filtration rate by CKD-EPI creatinine formulation ( $\mathrm{mL}$ per min per $1.73 \mathrm{~m}^{2}$ )

$\begin{array}{lll}\text { Mean (SD) } & 92.8(18.58) & 92.7(17.38)\end{array}$

Median

$92 \cdot 8(101 \cdot 0-123 \cdot 3)$

$93.9(102 \cdot 5-123 \cdot 5)$

Proteinuria by urinalysis (dipstick)

Grade $0 \quad 873(91 \%) \quad 430(90 \%)$

Grade $1 \quad 81(9 \%) \quad 44(9 \%)$

Grade $2 \quad 4(<1 \%) \quad 3(<1 \%)$

10 year probability of hip fracture by FRAX $(\%) \dagger \quad 0.16 \%(0.04-0.43) \quad 0.16 \%(0.04-0.47)$

10 year probability of major osteoporotic fracture by $\quad 1.92 \%(1 \cdot 20-3 \cdot 07) \quad 1.94 \%(1 \cdot 17-3 \cdot 27)$ $\operatorname{FRAX}(\%) \dagger$

Data are median (IQR) or $\mathrm{n}(\%)$, unless stated otherwise. Tenofovir alafenamide group assigned to receive a fixed once-a-day dose of $150 \mathrm{mg}$ elvitegravir, $150 \mathrm{mg}$ cobicistat, $200 \mathrm{mg}$ emtricitabine, and $10 \mathrm{mg}$ tenofovir alafenamide; the tenofovir disoproxil fumarate group was assigned to continue taking one of four previous regimens containing tenofovir disoproxil fumarate. CKD- CKD-EPI=chronic Kidney Disease Epidemiology Collaboration. *An individual patient could have more than one risk factor category. tFor patients younger than 40 years $(n=666 ; 436$ patients in the tenofovir alafenamide group and 230 patients in tenofovir disoproxil fumarate group), the FRAX software calculated fracture probabilities at age 40 years.

Table 1: Baseline demographics and disease characteristics for the safety analysis set 
study was $3 \cdot 1 \%$ (SD $4 \cdot 76$ ); those assigned to the tenofovir alafenamide group $(\mathrm{n}=42)$ improved spine bone mineral density by $2 \cdot 83 \%$ (SD 4.76 ) whereas those remaining on previous treatment $(\mathrm{n}=16)$ had further reduction of $0 \cdot 74 \%$ (SD 3.55\%).

A greater number of patients in the tenofovir alafenamide group than in the tenofovir disoproxil fumarate group recovered from osteopenia or osteoporosis at either the hip or the spine during the 48 weeks $(\mathrm{p}<0 \cdot 0001$; figure 2). All reported fracture adverse events

\begin{tabular}{|c|c|c|c|}
\hline & $\begin{array}{l}\text { Tenofovir } \\
\text { alafenamide } \\
\text { group }\end{array}$ & $\begin{array}{l}\text { Tenofovir } \\
\text { disoproxil } \\
\text { fumarate group }\end{array}$ & $\begin{array}{l}\text { Difference in least square } \\
\text { means* }(95 \% \mathrm{Cl})\end{array}$ \\
\hline \multicolumn{4}{|l|}{ Hip BMD change } \\
\hline Number assessed & 869 & 428 & .. \\
\hline BMD T-score change from baseline & $0.11(0.18)$ & $-0.02(0.20)$ & $0.13(0.10-0.15) ; p<0.0001$ \\
\hline Percent change from baseline & $1 \cdot 47 \%(2 \cdot 71)$ & $-0 \cdot 34 \%(2 \cdot 83)$ & $1.81(1.49-2.13) ; p<0.0001$ \\
\hline \multicolumn{4}{|l|}{ Patients with: } \\
\hline $0-3 \%$ increase & $486(56 \%)$ & $163 / 428(38 \%)$ & $p<0.0001 \dagger$ \\
\hline$>3 \%$ increase & $186 / 869(21 \%)$ & $32(8 \%)$ & .. \\
\hline \multicolumn{4}{|l|}{ Spine BMD change } \\
\hline Number assessed & 881 & 436 & \\
\hline BMD T-score change from baseline & $0.17(0.29)$ & $-0.02(0.31)$ & $0.19(0.16-0.23) ; p<0.0001$ \\
\hline Percent change from baseline & $1 \cdot 56 \%(3 \cdot 84)$ & $-0 \cdot 44 \%(4 \cdot 14)$ & $2.00(1.55-2.45) ; p<0.0001$ \\
\hline \multicolumn{4}{|l|}{ Patients with: } \\
\hline $0-3 \%$ increase & $358 / 881(41 \%)$ & $146 / 436(34 \%)$ & $\mathrm{p}<0.0001 \dagger$ \\
\hline$>3 \%$ increase & 291/881 (33\%) & $58 / 436(13 \%)$ & .. \\
\hline \multicolumn{4}{|c|}{$\begin{array}{l}\text { Data are } \mathrm{n} / \mathrm{N} \text { (\%) or mean (SD). Tenofovir alafenamide group assigned to receive a fixed once-a-day dose of } 150 \mathrm{mg} \\
\text { elvitegravir, } 150 \mathrm{mg} \text { cobicistat, } 200 \mathrm{mg} \text { emtricitabine, and } 10 \mathrm{mg} \text { tenofovir alafenamide; the tenofovir disoproxil } \\
\text { fumarate group was assigned to continue taking one of four previous regimens containing tenofovir disoproxil } \\
\text { fumarate. } \mathrm{BMD}=\text { bone mineral density. }{ }^{*} \text { Difference in least squares means and the } 95 \% \mathrm{Cl} \text { were calculated with analysis } \\
\text { of variance model (ANOVA) in which current treatment and prior treatment were fixed effects; } \mathrm{p} \text { value from the } \\
\text { Cochran-Mantel-Haenszel test. } \dagger^{\mathrm{p}} \text { value from the Cochran-Mantel-Haenszel test. }\end{array}$} \\
\hline
\end{tabular}

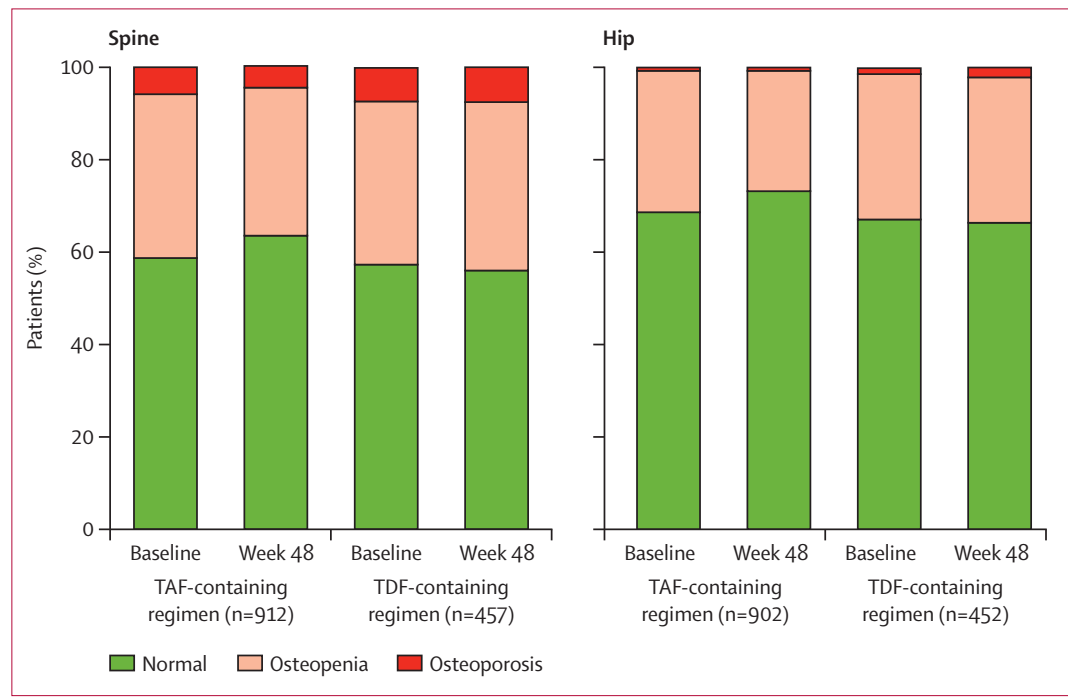

Figure 2: Changes in osteopenia and osteoporosis (T-score defined) from baseline to week 48

Differences between regimens were significant $(\mathrm{p}<0.0001)$. TAF=tenofovir alafenamide. TDF=Tenofovir disoproxil fumarate were the result of trauma and considered unrelated to treatment; no fragility fractures were reported.

Excluding patients switched from efavirenz, emtricitabine, and tenofovir disoproxil fumarate (an unboosted regimen), mean serum creatinine in those assigned to the tenofovir disoproxil fumarate group rose compared with the tenofovir alafenamide $(2.9 \mu \mathrm{mol} / \mathrm{L}$ [SD 9.29] vs $-0.4 \mu \mathrm{mol} / \mathrm{L}$ [10.14] in the tenofovir alafenamide group; difference in least squares mean for tenofovir alafenamide group versus tenofovir disoproxil fumarate group $-3.33 \mu \mathrm{mol} / \mathrm{L}, 95 \% \mathrm{CI}-4.57$ to $-2 \cdot 10 \mu \mathrm{mol} / \mathrm{L} ; \mathrm{p}<0 \cdot 0001)$. In patients who switched from efavirenz, emtricitabine, and tenofovir disoproxil fumarate to elvitegravir, cobicistat, emtricitabine, and tenofovir alafenamide, mean serum creatinine increased by $9 \cdot 2 \mu \mathrm{mol} / \mathrm{L}$ consistent with the established cobicistat effect on inhibition of serum creatinine excretion, ${ }^{22}$ whereas patients remaining on efavirenz, emtricitabine, and tenofovir disoproxil fumarate had a mean increase of $1.77 \mu \mathrm{mol} / \mathrm{L}$. eGFR $\mathrm{CG}_{\mathrm{CG}}$ values increased in the tenofovir alafenamide group (median $1.2 \mathrm{~mL}$ per min, IQR -6.6 to 9.1) compared with decreases from baseline in the tenofovir disoproxil fumarate group $(-3.7 \mathrm{~mL}$ per min, IQR $-10 \cdot 5$ to $3 \cdot 5)$ between week 2 and week 48 ( $<<0 \cdot 0001)$ after excluding patients on efavirenz, emtricitabine, and tenofovir disoproxil fumarate pre-randomisation.

General proteinuria and proximal tubular proteinuria decreased in patients assigned to the tenofovir alafenamide group, irrespective of previous treatment regimen, with significant differences between the treatment arms beginning at week 2 . By contrast, patients in the tenofovir disoproxil fumarate group had increases in each of these tests of proteinuria, with significant differences between the groups noted throughout and at week $48(\mathrm{p}<0 \cdot 001$; appendix).

The tenofovir alafenamide regimen was well tolerated through a median of 82.9 weeks (IQR 72.1-84.3) of exposure, with nine $(1 \%)$ patients discontinuing the drug due to adverse events and no drug-related serious adverse events (table 3). Patients in the tenofovir alafenamide group had a higher incidence of drug-related adverse events than did those in the tenofovir disoproxil fumarate group ( $21 \%$ vs $16 \%$ ), but the types of adverse events were similar between the groups (table 3).

Four patients died in the study, all in the tenofovir alafenamide group. One each died due to septic shock, stage 4 lung adenocarcinoma, and methamphetaminerelated myocarditis; one patient died from undetermined causes who had recent and substantial alcohol use; no deaths were considered treatment-related. Three patients in the tenofovir disoproxil fumarate group (all on boosted atazanavir) had jaundice leading to discontinuation. Kidney-related adverse events leading to treatment discontinuation were reported for two patients in the tenofovir alafenamide group and in five patients in the tenofovir disoproxil fumarate group. No cases of proximal tubulopathy or Fanconi's syndrome were reported ini the 
tenofovir alafenamide group; one patient from the tenofovir disoproxil fumarate group taking cobicistatboosted atazanavir, emtricitabine, and tenofovir disoproxil fumarate had Fanconi's syndrome and needed discontinuation.

Laboratory abnormalities were similar in both treatment groups. Grade 3 or 4 abnormalities were noted in $244(25 \%)$ patients in the tenofovir alafenamide group and $150(31 \%)$ in the tenofovir disoproxil fumarate group (driven by the higher incidence of grade 3 or 4 hyperbilirubinaemia). Fasting lipid concentrations increased from baseline in the tenofovir alafenamide group but remained stable in the tenofovir disoproxil fumarate group (appendix). During the study, $76(8 \%)$ patients in the tenofovir alafenamide group and $28(6 \%)$ patients in the tenofovir disoproxil fumarate began lipid-lowering drugs.

\section{Discussion}

As far as we are aware, this was the largest randomised controlled switch study ever done in virologically suppressed patients with HIV. After establishing noninferiority of the tenofovir alafenamide regimen (elvitegravir $150 \mathrm{mg}$, cobicistat $150 \mathrm{mg}$, emtricitabine $200 \mathrm{mg}$, and tenofovir alafenamide $10 \mathrm{mg}$ ) to four tenofovir disoproxil fumarate-containing regimens at week 48 , this study also showed that switching to the open-label tenofovir alafenamide regimen was also superior to continuing with the tenofovir disoproxil fumarate containing regimens. Both treatment groups had similar rates of virological failure at week 48 , but more patients in the tenofovir disoproxil fumarate group had a last viral load less than 50 copies per $\mathrm{mL}$ and yet discontinued study drug due to other reasons.

Patients previously on a regimen of efavirenz, emtricitabine, and tenofovir disoproxil fumarate or boosted atazanavir, emtricitabine, and tenofovir disoproxil fumarate regimens had significant efficacy advantages when switched to the tenofovir alafenamide group. These advantages might have occurred because this was an open-label study: some patients might have been unwilling to tolerate efavirenz-related CNS sideeffects or atazanavir-related hyperbilirubinaemia. By contrast, patients who switched from tenofovir disoproxil fumarate to tenofovir alafenamide (remaining on elvitegravir, cobicistat, and emtricitabine) had similar rates of virological success at week 48 ( $98 \%$ vs $97 \%$ ).

Treatments were well tolerated in both groups. The numerically higher frequency of adverse event reports for those switched to the tenofovir alafenamide regimen might be due to reporting bias in this open-label study in conjunction with a selection bias for those who continued their previous treatment regimen and were already tolerating it. Nonetheless, switching to the tenofovir alafenamide group showed several important safety advantages of tenofovir alafenamide over any of the tenofovir disoproxil fumarate-containing regimens.
Patients on prior tenofovir disoproxil fumaratecontaining regimens had improvements in their hip and bone mineral density after switching to the tenofovir alafenamide regimen. Previous data show that HIV-1 infected patients who begin ART have a reduction in bone mineral density in the first 24 to 48 weeks after starting treatment ${ }^{23,24}$ and this has been shown to be of higher magnitude with the use of tenofovir disoproxil fumarate-containing regimens. ${ }^{6,25,26}$

Little data on bone mineral density exist for patients switching ART regimens. In the OsteoTDF study, 26 virologically suppressed patients switched from tenofovir disoproxil fumarate to abacavir and had hip density improve by $2 \cdot 1 \%$ and spine density decrease by $0 \cdot 7 \%$ after 48 weeks. ${ }^{27} 16$ virologically suppressed patients

\begin{tabular}{|c|c|c|}
\hline & $\begin{array}{l}\text { Tenofovir } \\
\text { alafenamide } \\
\text { group }(n=959)\end{array}$ & $\begin{array}{l}\text { Tenofovir } \\
\text { disoproxil } \\
\text { fumarate } \\
\text { group }(n=477)\end{array}$ \\
\hline Any adverse event & $828(86 \%)$ & $399(84 \%)$ \\
\hline Study drug-related adverse event & $204(21 \%)$ & $76(16 \%)$ \\
\hline Grade 3 or 4 adverse event & $84(9 \%)$ & $54(11 \%)$ \\
\hline Serious adverse event & $65(7 \%)$ & $35(7 \%)$ \\
\hline $\begin{array}{l}\text { Study drug-related serious adverse } \\
\text { event }\end{array}$ & 0 & $2(<1 \%)$ \\
\hline Premature study drug discontinuation & $9(1 \%)^{*}$ & $12(3 \%) \dagger$ \\
\hline \multicolumn{3}{|l|}{$\begin{array}{l}\text { Most common treatment-emergent } \\
\text { adverse event }\end{array}$} \\
\hline Upper respiratory tract infection & $151(16 \%)$ & $54(11 \%)$ \\
\hline Diarrhoea & $96(10 \%)$ & $42(9 \%)$ \\
\hline Nasopharyngitis & $88(9 \%)$ & $39(8 \%)$ \\
\hline Headache & $69(7 \%)$ & $20(4 \%)$ \\
\hline Cough & $64(7 \%)$ & $25(5 \%)$ \\
\hline Syphilis & $46(5 \%)$ & $30(6 \%)$ \\
\hline Insomnia & $50(5 \%)$ & $30(6 \%)$ \\
\hline Arthralgia & $59(6 \%)$ & $24(5 \%)$ \\
\hline Bronchitis & $58(6 \%)$ & $26(5 \%)$ \\
\hline Depression & $42(4 \%)$ & $30(6 \%)$ \\
\hline Osteopenia & $56(6 \%)$ & $22(5 \%)$ \\
\hline Back pain & $52(5 \%)$ & $25(5 \%)$ \\
\hline Nausea & $50(5 \%)$ & $16(3 \%)$ \\
\hline Sinusitis & $48(5 \%)$ & $25(5 \%)$ \\
\hline \multicolumn{3}{|c|}{$\begin{array}{l}\text { Data are n (\%). Tenofovir alafenamide group assigned to receive a fixed } \\
\text { once-a-day dose of } 150 \text { mg elvitegravir, } 150 \mathrm{mg} \text { cobicistat, } 200 \mathrm{mg} \text { emtricitabine } \\
\text { and } 10 \mathrm{mg} \text { tenofovir alafenamide; the tenofovir disoproxil fumarate group was } \\
\text { assigned to continue taking one of four previous regimens containing tenofovir } \\
\text { disoproxil fumarate. *Adverse event-related discontinuations in one patient each } \\
\text { from the tenofovir alafenamide group include: panic attack; memory loss, speech } \\
\text { disturbance, and lack of motivation; acute renal failure; Reiter's syndrome; } \\
\text { nausea, vomiting, and headache; suicide attempt; leg swelling and impaired } \\
\text { concentration; depression; and interstitial nephritis. †Adverse event-related } \\
\text { discontinuations in one patient each from the tenofovir disoproxil fumarate } \\
\text { group include: abnormal dreams; depression, insomnia, and irritability; } \\
\text { depression, insomnia, and nightmares; elevated bilirubin; icterus ( } n=2) \text {; increased } \\
\text { forgetfulness; chronic kidney disease; elevated serum creatinine; Fanconi's } \\
\text { syndrome and mild jaundice; increased creatinine; and nephritic colic. }\end{array}$} \\
\hline
\end{tabular}


with HIV switched from two nucleoside reverse transcriptase inhibitors plus a protease inhibitor to protease inhibitor monotherapy and had a $0.2 \%$ increase for hip density and $0.08 \%$ decrease for spine density after 1 year. ${ }^{28}$ In a study of 37 patients with T scores lower than -1.0 who were taking a boosted protease inhibitor, switching from tenofovir disoproxil fumarate to raltegravir increased bone mineral density in the hip by $2.5 \%$ and in the spine by $3.0 \%$ after 48 weeks. ${ }^{29}$ In this study of more than 1400 virologically suppressed patients, mean percentage change in hip and spine bone mineral density improved by $1.47 \%$ and $1.56 \%$ respectively. The findings in the current study provide helpful information on the potential pathophysiology of tenofovir disoproxil fumarate-related changes in bone mineral density because they represent the largest body of data in a single study; switching to the tenofovir alafenamide regimen led to statistically significant improvements irrespective of the third agent used with tenofovir disoproxil fumarate; switching to tenofovir alafenamide from tenofovir disoproxil fumarate while remaining on elvitegravir, cobicistat, and emtricitabine resulted in significant improvement of bone mineral density. Moreover, T-score status for both hip and spine significantly improved for those assigned to the tenofovir alafenamide group, and not to tenofovir disoproxil fumarate. Additional longer term follow up data are needed to assess the clinical relevance of these differences in bone mineral density.

The switch to tenofovir alafenamide also resulted in improvements in renal function, including decreases in serum creatinine (those switching from a boosted regimen), decreases in dipstick proteinuria, decreases in quantitative tests of total urine protein, and total urine albumin, decreases in specific proximal renal tubular proteins, and improvements in tests of proximal renal tubular function (fractional excretion of uric acid, fractional excretion of phosphate, and renal tubular maximum reabsorption rate of phosphate to the glomerular filtration rate; appendix). Proteinuria, albuminuria, and specific proximal tubular proteinuria have been shown to increase risk of mortality or cardiovascular events in both the general population and in HIV-1 infected individuals. ${ }^{30-34}$ By significantly reducing proteinuria, the switch to tenofovir alafenamide could affect mortality or cardiovascular events. Changes noted in renal laboratory tests occurred as early as week 2 after the switch from a tenofovir disoproxil fumarate-containing regimen and remained significantly different to week 48 . Although the exact mechanism for these improvements is not known, it could be related to the lower plasma tenofovir concentrations in patients switched to tenofovir alafenamide. ${ }^{6}$ Further, tenofovir is a substrate for transporters on the basolateral surface of proximal renal tubular cells (OAT1, OAT3) whereas tenofovir alafenamide is not. ${ }^{35}$ Therefore, although direct measurements were not made, patients switched to the tenofovir alafenamide-containing regimen probably had lower levels of tenofovir inside proximal renal tubular cells. Before entry into this study, all patients received at least 96 weeks of a tenofovir disoproxil fumarate-containing regimen. Chronic tenofovir disoproxil fumarate use can worsen kidney function, which is probably mediated through dysfunction in the proximal tubular cells. ${ }^{36-38}$ Although the renal safety implications for use of elvitegravir, cobicistat, emtricitabine, and tenofovir alafenamide in patients with pre-existing renal comorbidities are encouraging, the clinical relevance of improved renal parameters for patients who switched to tenofovir alafenamide regimens needs longer-term follow-up.

Increases in fasting total cholesterol, fasting LDL cholesterol, and fasting triglycerides were noted in the tenofovir alafenamide group, consistent with clinical data in patients who switch to a non-tenofovir disoproxil fumarate-containing ART regimen. Nonetheless, the total cholesterol to HDL ratio had only minimal changes from baseline. Compared with other regimens, use of tenofovir disoproxil fumarate has been linked to reduced lipids in treatment-naive patients than with other nucleoside reverse transcriptase inhibitors, ${ }^{39,40}$ potentially due to higher tenofovir concentrations in plasma. In this study and in two blinded and controlled studies in treatment-naive patients, patients on tenofovir alafenamide had slightly higher lipid concentrations than had those on tenofovir disoproxil fumarate. ${ }^{41,42}$ Reported frequency of lipid-related or cardiovascular adverse events were similar between the two groups.

Limitations to these data do exist. This was an openlabel study in patients switching from a treatment regimen they were tolerating, and so caution is warranted in interpretation of the between-group differences in adverse event-related discontinuations and in subjective safety reports. Longer-term follow-up might be needed to understand the clinical advantages associated with the improvements in bone and renal safety observed in those switching to tenofovir alafenamide.

In conclusion, virologically suppressed HIV-1-infected patients who switched to a tenofovir alafenamidecontaining regimen (elvitegravir, cobicistat, emtricitabine, and tenofovir alafenamide) maintained virological suppression at a statistically higher rate at 48 weeks compared with those who remained on one of four tenofovir disoproxil fumarate-containing regimens (elvitegravir, cobicistat, emtricitabine, and tenofovir disoproxil fumarate; efavirenz, emtricitabine, and tenofovir disoproxil fumarate; cobicistat-boosted atazanavir plus emtricitabine and tenofovir disoproxil fumarate; or ritonavir-boosted atazanavir plus emtricitabine and tenofovir disoproxil fumarate). The tenofovir alafenamide regimen was well tolerated and patients had significant improvements in bone and renal safety. Virologically supressed patients can be switched from tenofovir disoproxil fumarate-containing regimens to regimens containing tenofovir alafenamide without a loss of efficacy. 
Virologically suppressed patients can be switched from a broad range of tenofovir disoproxil fumarate-containing regimens to a regimen containing tenofovir alafenamide without loss of efficacy.

\section{Contributors}

AM enrolled patients and edited and approved the report. JRA, JA-V, GD JVL, EK, RE, MC, JVM, JB, DS, ED, CO, DAW, IB, JLS, P-MG, and GH enrolled patients, reviewed and interpreted data, and edited drafts of the report. Y-PL, AKC, and SM designed the study. AP, Y-PL, AKC, and SM oversaw data collection. Y-PL, AKC, and SM analysed data, and provided interpretation. AM, Y-PL, and SM wrote the first draft of the report. All authors contributed to edits of the final manuscript and SM served as the corresponding author.

\section{Declaration of interests}

AM received grants, personal fees and non-financial support from Gilead Sciences, grants and personal fees from ViiV, Janssen, and Merck, and grants from Bristol-Myers Squibb. JRA has received personal fees from Gilead Sciences, ViiV, Janssen, Abbvie, BristolMyers Squibb, and Merck. GD has received personal fees from Abbvie, grants and personal fees from Bristol-Myers Squibb, Gilead Sciences, Janssen, ViiV, and Merck. JVL has received grants from Gilead Sciences and began employment at ViiV Healthcare on July 15, 2015. RE has received grants, personal fees, and other support from Gilead Sciences, ViiV, and Janssen; RE has received grants and personal fees from Merck. MC has received grants from Bristol-Myers Squibb, Gilead Sciences, and ViiV. JVM has received honoraria for participation in advisory boards, conducted lectures, and conducted clinical trials for Abbott, Gilead Sciences, ViiV, Merck, Pfizer, and Roche. JB has received personal fees from Gilead Sciences. DS has received personal fees from Gilead Sciences, ViiV, Merck, and BristolMyers Squibb. ED has received personal fees from Gilead Sciences and Janssen. CO has received grants, personal fees, non-financial support, and other support from Gilead Sciences, ViiV, Bristol-Myers Squibb, and Abbvie. DAW has participated in advisory boards for Gilead Sciences. IB has received grants and other support from Gilead Sciences. JLS has received grants from Gilead Sciences. P-MG has received grants from Bristol-Myers Squibb and Janssen, personal fees from Bristol-Myers Squibb, and financial fees from Gilead, ViiV, and Abbvie to participate in international advisory boards. GH has received grants from Gilead Sciences, ViiV, Janssen, and Merck, and has participated on an advisory board for Biocryst. AP, Y-PL, AKC, and SM are employees of the sponsor, Gilead Sciences. JA-V and EK declare no competing interests.

\section{Acknowledgments}

We thank the contributions made to this study by all patients, their partners and families, and the investigators and their study teams. We also thank Marshall W Fordyce, Christian Callebaut, Lijie Zhong, Jay Huang, Keerthi Kosuri, Heena Patel and the GS-US-292-0109 study team for significant support of this study. Kris Dalmacio provided invaluable technical expertise in the preparation of the manuscript. Parts of this study were presented at the 8th IAS Conference on HIV Pathogenesis and Treatment; Vancouver, Canada; July 19-22, 2015.

\section{References}

1 Panel on Antiretroviral Guidelines for Adult and Adolescents. Guidelines for the use of antiretroviral agents in HIV-1 infected adults and adolescents. 2015. https://aidsinfo.nih.gov/contentfiles/ lvguidelines/adultandadolescentgl.pdf (accessed May 15, 2015).

2 Gupta SK. Tenofovir-associated Fanconi syndrome: review of the FDA adverse event reporting system. AIDS Patient Care STDS 2008; 22: 99-103.

3 Mocroft A, Kirk O, Reiss P, et al. Estimated glomerular filtration rate, chronic kidney disease and antiretroviral drug use in HIV-positive patients. AIDS 2010; 24: 1667-78.

4 McComsey GA, Kitch D, Daar ES, et al. Bone mineral density and fractures in antiretroviral-naive persons randomized to receive abacavir-lamivudine or tenofovir disoproxil fumarate-emtricitabine along with efavirenz or atazanavir-ritonavir: AIDS Clinical Trials Group A5224s, a substudy of ACTG A5202. J Infect Dis 2011; 203: 1791-801.
5 Bedimo R, Maalouf NM, Zhang S, Drechsler H, Tebas P. Osteoporotic fracture risk associated with cumulative exposure to tenofovir and other antiretroviral agents. AIDS 2012; 26: 825-31.

6 Sax PE, Wohl D, Yin MT, et al. Tenofovir alafenamide versus tenofovir disoproxil fumarate, coformulated with elvitegravir cobicistat, and emtricitabine, for initial treatment of HIV-1 infection: two randomised, double-blind, phase 3 , non-inferiority trials. Lancet 2015; 385: 2606-15.

7 Wojewoda CM, Spahlinger T, Harmon ML, et al. Comparison of Roche Cobas AmpliPrep/Cobas Taqman HIV-1 test version 2.0 (CAP/CTM v2.0) with other real-time PCR assays in HIV-1 monitoring and follow-up of low-level viral loads. J Virol Meth 2013; 187: 1-5.

8 Cockcroft DW, Gault MH. Prediction of creatinine clearance from serum creatinine. Nephron 1976; 16: 31-41.

9 Mocroft A, Ryom L, Reiss P, et al. A comparison of estimated glomerular filtration rates using Cockcroft-Gault and the Chronic Kidney Disease Epidemiology Collaboration estimating equations in HIV infection. HIV Med 2014; 15: 144-52.

10 Murata K, Baumann NA, Saenger AK, Larson TS, Rule AD, Lieske JC. Relative performance of the MDRD and CKD-EPI equations for estimating glomerular filtration rate among patients with varied clinical presentations. Clin J Am Soc Nephrol 2011; 6: 1963-72

11 Ibrahim F, Hamzah L, Jones R, Nitsch D, Sabin C, Post FA. Comparison of CKD-EPI and MDRD to estimate baseline renal function in HIV-positive patients. Nephrol Dial Transplant 2012; 27: 2291-97.

12 US Department of Health and Human Services Food and Drug Administration, Center for Drug Evaluation and Research. Guidance for industry. Human immunodeficiency virus-1 infection: developing antiretroviral drugs for treatment. June, 2013. http://www.fda.gov/downloads/drugs/ guidancecomplianceregulatoryinformatio/guidances/ucm 355128 pdf (accessed Feb 2, 2015).

13 Hodder SL, Mounzer K, Dejesus E, et al. Patient-reported outcomes in virologically suppressed, HIV-1-Infected subjects after switching to a simplified, single-tablet regimen of efavirenz, emtricitabine, and tenofovir DF. AIDS Patient Care STDS 2010; 24: 87-96.

14 Wu AW, Hanson KA, Harding G, et al. Responsiveness of the MOS-HIV and EQ-5D in HIV-infected adults receiving antiretroviral therapies. Health Qual Life Outcomes 2013; 11: 42.

15 Hsiung PC, Fang CT, Chang YY, Chen MY, Wang JD. Comparison of WHOQOL-bREF and SF-36 in patients with HIV infection. Qual Life Res 2005; 14: 141-50.

16 Bristol-Myers Squibb. Sustiva (efavirenz) package insert. 2015 http://packageinserts.bms.com/pi/pi_sustiva.pdf (accessed July 15, 2015).

17 Marzolini C, Telenti A, Decosterd LA, Greub G, Biollaz J, Buclin T. Efavirenz plasma levels can predict treatment failure and central nervous system side effects in HIV-1-infected patients. AIDS 2001; 15: 71-75.

18 Clifford DB, Evans S, Yang Y, et al. Impact of efavirenz on neuropsychological performance and symptoms in HIV-infected individuals. Ann Intern Med 2005; 143: 714-21.

19 McCloskey DE. FRAX® Identifying people at high risk of fracture. 2009. http://www.iofbonehealth.org/sites/default/files/PDFs/ WOD\%20Reports/FRAX_report_09.pdf (accessed July 15, 2015)

20 Koch GG, Carr GJ, Amara IA, et al. Categorical data analysis. In: Berry DA, ed. Statistical methodology in the pharmaceutical sciences. New York, NY: Marcel Dekker, 1989: 21-41.

21 Clumeck N, Molina JM, Henry K, et al. A randomized, double-blind comparison of single-tablet regimen elvitegravir/cobicistat/ emtricitabine/tenofovir DF vs ritonavir-boosted atazanavir plus emtricitabine/tenofovir DF for initial treatment of HIV-1 infection: analysis of week 144 results. J Acquir Immune Defic Syndr 2014; 65: e121-24.

22 German P, Liu HC, Szwarcberg J, et al. Effect of cobicistat on glomerular filtration rate in subjects with normal and impaired renal function. J Acquir Immune Defic Syndr 2012; 61: 32-40.

23 Tebas P, Powderly WG, Claxton S, et al. Accelerated bone mineral loss in HIV-infected pateints receiving potent antiretroviral therapy. AIDS 2000; 14: F63-67. 
24 Hansen AB, Obel N, Nielsen H, Pedersen C, Gerstoft J. Bone mineral density changes in protease inhibitor-sparing vs. nucleoside reverse transcriptase inhibitor-sparing highly active antiretroviral therapy. HIV Med 2011; 12: 157-65.

25 Stellbrink H-J, Orkin C, Arribas JR, et al. Comparison of changes in bone density and turnover with abacavir-lamivudine versus tenofovir-emtricitabine in HIV-infected adults: 48-week results from the ASSERT study. Clin Infect Dis 2010; 51: 963-72.

26 Rockstroh JK, DeJesus E, Henry K, et al. A randomized double-blind comparison of coformulated elvitegravir/cobicistat/ emtricitabine/tenofovir DF vs ritonavir-boosted atazanavir plus coformulated emtricitabine and tenofovir DF for initial treatment of HIV-1 infection: analysis of week 96 results. J Acquir Immune Defic Syndr 2013; 62: 483-86.

27 Negredo E, Domingo P, Perez-Alvarez N, et al. Improvement in bone mineral density after switching from tenofovir to abacavir in HIV-1-infected patients with low bone mineral density: two-centre randomized pilot study (OsteoTDF study). J Antimicrob Chemother 2014; 69: 3368-71.

28 Negredo E, Bonjoch A, Puig J, et al. Long-term changes in bone mineral density after switching to a protease inhibitor monotherapy in HIV-infected subjects. New Microbiol 2015; 38: 193-99.

29 Bloch M, Tong WWY, Hoy J, et al. Switch from tenofovir to raltegravir increases low bone mineral density and decreases markers of bone turnover over 48 weeks. HIV Med 2014; 15: 373-80

30 Schrader J, Luders S, Kulschewski A, et al. Microalbuminuria and tubular proteinuria as risk predictors of cardiovascular morbidity and mortality in essential hypertension: final results of a prospective long-term study (MARPLE Study). J Hypertens 2006; 24: $541-48$

31 George E, Lucas GM, Nadkarni GN, Fine DM, Moore R, Atta MG Kidney function and the risk of cardiovascular events in HIV-1 infected patients. AIDS 2010; 24: 387-94.

32 Wyatt CM, Hoover DR, Shi Q, et al. Microalbuminuria is associated with all-cause and AIDS mortality in women with HIV infection. J Acquir Immune Defic Syndr 2010; 55: 73-77.
33 Mulenga LB, Kruse G, Lakhi S, et al. Baseline renal insufficiency and risk of death among HIV-infected adults on antiretroviral therapy in Lusaka, Zambia. AIDS 2008; 22: 1821-27.

34 Gardner LI, Holmberg SD, Williamson JM, et al. Development of proteinuria or elevated serum creatinine and mortality in HIV-infected women. J Acquir Immune Defic Syndr 2003; 32: 203-09.

35 Bam RA, Yant SR, Cihlar T. Tenofovir alafenamide is not a substrate for renal organic anion transporters (OATs) and does not exhibit OAT-dependent cytotoxicity. Antivir Ther 2014; 19: 687-92.

36 Hall AM, Hendry BM, Nitsch D, Connolly JO. Tenofovir-associated kidney toxicity in HIV-infected patients. Am J Kidney Dis 2011; 57: 773-80.

37 Nisjijima T, Kawasaki Y, Tanaka N, et al. Long-term exposure to tenofovir continuously decrease renal function in HIV-1-infected patients with low body weight: results from 10 years of follow-up. AIDS 2014; 28: 1903-10.

38 Kinai E, Hanabusa H. Progressive renal tubular dysfunction associated with long-term use of tenofovir DF. AIDS Res Hum Retroviruses 2009; 25: 387-94.

39 Tungsiripat M, Kitch D, Glesby MJ, et al. A pilot study to determine the impact on dyslipidemia of adding tenofovir to stable background antiretroviral therapy: ACTG 5206. AIDS 2010; 24: 1781-84.

40 Souza SJ, Luzia LA, Santos SS, Carvalho Rondo PH. Lipid profile of HIV-infected patients in relation to antiretroviral therapy: a review. Rev Ass Med Bras 2013; 59: 186-98.

41 Gallant JE, Staszewski S, Pozniak AL, et al. Efficacy and safety of tenofovir DF vs stavudine in combination therapy in antiretroviral-naive patients: a 3-year randomized trial. JAMA 2004; 292: 191-201.

42 Crane HM, Grunfeld C, Willig JH, et al. Impact of NRTIs on lipid levels among a large HIV-infected cohort initiating antiretroviral therapy in clinical care. AIDS 2011; 25: 185-95. 